\title{
Ingestão de Cálcio e Vitamina D e Risco de Câncer Colorretal: uma Revisão Bibliográfica
}

\author{
Calcium and Vitamin D Intake and Colorectal Cancer Risk: a Bibliographic \\ Review \\ Ingesta de Calcio y Vitamina D y el Riesgo de Cáncer Colorrectal: Revisión \\ Bibliografía
}

\author{
Claudia Merlo Cabral', Nádia Dias Gruezo²
}

\section{Resumo}

O câncer colorretal apresenta-se como o terceiro tipo de neoplasia mais prevalente de câncer em todo o mundo. Os principais fatores de risco são: histórico familiar de câncer de cólon e reto, dieta com base em gorduras animais, baixa ingestão de frutas, vegetais e cereais, consumo excessivo de álcool e fumo, e idade avançada. Alguns estudos em animais têm evidenciado uma possível atuação do cálcio e da vitamina $\mathrm{D}$ como anticarcinógenos colorretal. O objetivo é discutir a associação entre ingestão alimentar e suplementação de cálcio, vitamina $\mathrm{D}$ e risco de câncer colorretal. Realizou-se uma revisão bibliográfica, de artigos publicados nos últimos dez anos, de fontes de revistas indexadas em bibliotecas virtuais Lilacs, SciELO e Medline. Um dos mecanismos pelo qual o cálcio reduz o risco do câncer colorretal é a capacidade do cálcio em se ligar aos sais biliares e aos ácidos graxos ionizados, reduzindo o efeito destes compostos de proliferação celular da mucosa colônica. A vitamina D tem uma ação que regula a proliferação celular. Estudos mostraram que o alto consumo de produtos lácteos, principalmente o leite, está associado à diminuiçáo do risco. Existem evidências de que o cálcio e vitamina $\mathrm{D}$ estão relacionados com redução da gênese do câncer colorretal.

Palavras-chave: Neoplasias Colorretais; Cálcio; Vitamina D; Laticínios; Revisão

${ }^{1}$ Especialista em Nutrição Oncológica pelo Instituto Nacional de Câncer (INCA). Especialista em Nutrição Clínica pela Faculdade Federal Fluminense (UFF). Residência em Nutrição Clínica/Hospital Universitário Pedro Ernesto/Universidade Estadual do Rio de Janeiro (UERJ). Nutricionista da Fundação Municipal Hospitalar de Macaé (RJ), Brasil.

${ }^{2}$ Mestre em Saúde da Família da Universidade Estácio de Sá (UNESA/RJ). Especialista em Nutrição Oncológica pelo INCA. Supervisora de Nutrição Clínica do Serviço de Nutrição e Dietética do Hospital do Câncer I (HC I)/ INCA.

Endereço para correspondência: E-mail: merlo.c@hotmail.com 


\section{INTRODUÇÃO}

O câncer configura-se como um dos principais problemas de saúde pública mundial. É uma doença crônico-degenerativa que afeta várias dimensóes da vida humana e causa importante impacto econômico na sociedade, necessitando de tratamento especializado prolongado e oneroso. Além disso, é responsável pela redução do potencial de trabalho humano e perda de muitas vidas. Para o ano de 2020, são esperados mais de 15 milhóes de casos novos de câncer no mundo ${ }^{1}$.

No mundo, o câncer colorretal representa a terceira causa mais comum de câncer em ambos os sexos e a segunda em países desenvolvidos. Os padróes geográficos são bem similares entre homens e mulheres, porém o câncer de reto é cerca de $20 \%$ a $50 \%$ maior em homens na maioria das populaçóes. O número de casos novos de câncer de cólon e reto estimados para o Brasil no ano de 2010 é de 13.310 casos em homens e de 14.800 em mulheres. Esses valores correspondem a um risco estimado de 14 casos novos a cada 100 mil homens e 15 para cada 100 mil mulheres ${ }^{2}$.

A sobrevida para esse tipo de neoplasia é considerada boa se a doença for diagnosticada em estádio inicial. A sobrevida média global em cinco anos varia entre $40 \%$ a $50 \%$, não sendo observadas grandes diferenças entre países desenvolvidos e países em desenvolvimento. Esse relativo bom prognóstico faz com que o câncer de cólon e reto seja o segundo tipo de câncer mais prevalente em todo o mundo com aproximadamente 2,4 milhóes de pessoas vivas diagnosticadas com essa neoplasia. $O$ fator de risco mais importante para esse tipo de neoplasia é a história familiar de câncer de cólon e reto e a predisposição genética ao desenvolvimento de doenças crônicas do intestino, como as poliposes adenomatosas; além de uma dieta com base em gorduras animais, baixa ingestão de frutas, vegetais e cereais, assim como consumo excessivo de álcool e tabagismo. A prática de atividade física regular está associada a um baixo risco de desenvolvimento do câncer de cólon e reto; além disso, a idade também é considerada um fator de risco, uma vez que tanto a incidência como a mortalidade aumentam com o aumento da idade ${ }^{2}$.

A dieta é um dos fatores mais importantes para o desenvolvimento de câncer colorretal, porém componentes específicos da dieta não têm sido bem estudados. Atualmente, sabe-se que os fatores dietéticos que são relacionados com a carcinogênese no intestino grosso são: excesso de gordura animal, carne vermelha e calorias; baixa ingestão de fibras e, provavelmente, o álcool e fumo. Em contrapartida, alto consumo de frutas, vegetais frescos, cereais e peixe, baixo consumo de carnes vermelhas e processadas, e de bebidas alcoólicas, bem como a prática de atividade física, estão associados a um menor risco de desenvolvimento de câncer colorretal ${ }^{3}$.

Estudos evidenciam uma possível atuação do cálcio e da vitamina $\mathrm{D}$ como anticarcinógenos no cólon e reto ${ }^{4}$.

Uma das hipóteses do cálcio para redução do risco do câncer colorretal é a capacidade do cálcio em se ligar aos sais biliares e aos ácidos graxos ionizados, reduzindo o efeito desses compostos de proliferaçáo celular da mucosa colonica ${ }^{5}$. Outra hipótese descrita consiste na atuação do cálcio promovendo a diferenciação de células colônicas através da conexão de receptores sensíveis ao cálcio, resultando em várias açóes biológicas tais como a ativação da proteína $\mathrm{C}$ quinase. Acredita-se que a vitamina $\mathrm{D}$, que exerce uma ação de regulação de proliferação celular, em sua forma mais ativa, 1,25-dihidroxivitamina D, foi apontada como regulador da proliferação e diferenciação de células cancerígenas de humanos ${ }^{5}$.

Este estudo tem como objetivo descrever a relação entre a ingestão alimentar e suplementação de cálcio e vitamina D com o risco do câncer colorretal.

\section{METODOLOGIA}

Realizou-se uma revisão bibliográfica na qual foram utilizadas as bases de dados on-line: Medline, Lilacs e SciELO.

Os artigos pesquisados foram publicados entre 1997 a setembro de 2009 nas línguas inglesa, espanhola ou portuguesa.

Para a busca, utilizaram-se as seguintes palavras-chave: colorretal cancer, calcium, vitamin $D$, dairy food e obteve-se um total de 25 referências.

Para a seleção dos artigos, foram adotados os seguintes critérios de inclusão: tratar-se preferencialmente de câncer colorretal e discutir sobre ingestão de cálcio e vitamina D.

\section{RESULTADOS E DISCUSSÃO}

Dezessete artigos atenderam a esses critérios e todos foram recuperados e incluídos neste estudo. Outras obras $(n=8)$ foram incluídas a partir das referências bibliográficas contidas nos artigos seguindo a metodologia supracitada.

\section{INGESTÃO E SUPLEMENTAÇÃO DE CÁLCIO E VITAMINA D E RISCO DE CÂNCER COLORRETAL}

A vitamina $\mathrm{D}$ no fígado é hidroxilada para formar a 25-hidroxivitamina D $[25(\mathrm{OH}) \mathrm{D}]$, a principal forma circulante da vitamina D. No rim, a enzima $25(\mathrm{OH})$ D3-1-hidroxilase catalisa uma segunda hidroxilação da $25(\mathrm{OH}) \mathrm{D}$, resultando na formação de 1 alpha, 25dihidroxivitamina $\mathrm{D}$ [1,25(OH)2D] - a forma ativa da 
vitamina D. Esta tem a ação de inibir a proliferação e estimular a diferenciação das células 5 .

Em relação ao cálcio, há também evidências consideráveis de que ele, ao se ligar aos ácidos biliares e às gorduras, reduziria o conteúdo tóxico intraluminal diminuindo assim a proliferação de células do epitélio intestino grosso 5 .

Estudos sobre a expressão específica tecidual do CYP27B1-codificado, 25-hidroxivitamina D-1 $\alpha$ hidroxilase e do receptor de cálcio extracelular (CaR) esclareceram como a produçáo local de 1,25-D3 dihidroxivitamina $(1,25(\mathrm{OH}) 2 \mathrm{D} 3)$ e Ca $^{2+}$ extracelular conjuntamente seriam reguladores da proliferação, diferenciação e função celular. Verificou-se que a 1,25 $(\mathrm{OH})$ 2D3 e o cálcio interagem na modulação do crescimento celular em diferentes formas: sinalizam o caminho para o receptor da vitamina $\mathrm{D}$ (VDR) e o receptor extracelular de cálcio $(\mathrm{CaR})$ convergirem na mesma via de elementos; o alto nível de cálcio extracelular modularia o metabolismo extrarenal da vitamina $\mathrm{D}$ favorecendo um aumento das concentraçôes de 1,25(OH) 2D3; e ainda a $1,25(\mathrm{OH}) 2 \mathrm{D} 3$ regularia aumentando a expressão do $\mathrm{CaR}$ mediando respostas antiproliferativas de $\mathrm{Ca}^{2+}$ extracelular. Isso poderia explicar porque a suplementação de cálcio e vitamina $\mathrm{D}$ combinada seria necessária para a prevenção do câncer colorretal ${ }^{6}$.

Estudos epidemiológicos têm encontrado associação entre o consumo de produtos lácteos e Vitamina D e risco de câncer colorretal ${ }^{7}$ (Quadro 1).

Através de uma meta-análise de dez estudos coortes da América do Norte e da Europa, Cho et al. ${ }^{8}$ examinaram a associação entre o consumo de produtos lácteos e cálcio e risco de câncer colorretal, a qual incluiu 534.536 indivíduos com câncer colorretal. Os resultados mostraram que a ingestáo $>250 \mathrm{~g} /$ dia de leite estava relacionada com um menor risco de câncer colorretal quando comparado com uma ingestão $<70 \mathrm{~g} / \mathrm{dia}$, e que o consumo de $500 \mathrm{~g} /$ dia (aproximadamente 2 copos de leite) estava associado com a reduçáo em $12 \%$ do risco de câncer colorretal.

Larsson et al. ${ }^{9}$, por meio de um estudo coorte com participação de 45.306 homens sem história de câncer, realizado no período de 1997 a dezembro 2004 na Suécia, sustentaram a hipótese de que alta ingestão de cálcio e produtos lácteos, em especial o leite, estava relacionada com a diminuiçáo do risco de câncer colorretal. Além disso, observou que homens que consumiam $\geq 1,5$ copos de leite dia tinham 33\% menos risco de desenvolver câncer colorretal quando comparados com quem consumia $<2$ copos semana.

Corroborando os resultados do estudo anterior, um estudo coorte prospectivo com duração de 24 anos, com participação de 9.959 indivíduos de ambos os sexos, a partir de 15 anos de idade, sem história prévia de câncer, sugeriu que o consumo de leite e produtos lácteos em geral estava inversamente relacionado com a incidência do câncer colorretal. Além disso, constatou que indivíduos que estavam no maior quartil de consumo de leite tinham 46\% menos chance de desenvolver câncer de cólon e os que consumiam produtos láteos, $37 \%$ menos chance. Entretanto essa associação não aparece para ingestão total de cálcio, vitamina $\mathrm{D}$ ou leite fermentado ${ }^{10}$.

Hubner et al. ${ }^{11}$ analisaram a ingestáo dietética de cálcio, vitamina $\mathrm{D}$ e produtos lácteos de 853 participantes de um estudo de prevenção de adenoma colorretal e observou que o consumo de produtos lácteos estava inversamente relacionado com a reincidência de adenoma colorretal independente do consumo de vitamina D e suplementação de cálcio.

Cho et $a .^{8}$ analisaram o consumo de produtos lácteos, tais como: queijo cottage ou ricota, manteiga, creme ou sorvete de creme com risco de câncer colorretal e os participantes que consumiam mais que $25 \mathrm{~g} / \mathrm{dia}$ (categoria de alta ingestão) de queijo cottage e ricota tiveram um menor risco quando comparados com uma categoria de baixo consumo, entretanto outros produtos lácteos náo resultaram em uma reduçáo significativa do câncer colorretal.

Ao avaliar a ingestão de cálcio e cálcio total (cálcio dietético e suplementos), Cho et al. ${ }^{8}$ observaram que ambos estavam associados com um menor risco de câncer colorretal. Resultado semelhante foi observado no estudo de Larsson et al. ${ }^{9}$, no qual homens no maior quartil de ingestão de cálcio tinham menos risco para desenvolver câncer colorretal quando comparado com o menor quartil.

O II Estudo Coorte Nutricional de Prevenção de Câncer, realizado nos Estados Unidos, no período de 1992 a 1993, com participação de 66.882 mulheres e 60.866 homens, mostrou que houve uma modesta associação inversa entre ingestão total de cálcio nos homens, mas não nas mulheres. Contudo sugeriu que doses moderadas de cálcio reduzem o risco, mas altas doses não aumentariam esses benefícios ${ }^{12}$.

Em um estudo coorte, realizado durante 11,3 anos com 61,463 mulheres, foram observados 572 casos novos de câncer colorretal. Através de um questionário com 67 itens alimentares, eles observaram uma associação inversa entre a ingestão de cálcio e câncer colorretal. Mulheres com alta ingestão de cálcio (média de $914 \mathrm{mg} /$ dia) tiveram redução do risco de câncer colorretal quando comparadas com mulheres com baixa ingestão $(486 \mathrm{mg} / \mathrm{dia})$. A ingestão de produtos lácteos foi fraca, sugerindo que a ingestão de cálcio é mais importante que uma fonte específica de cálcio. A ingestão da vitamina $\mathrm{D}$ não estava claramente associada com o risco ${ }^{13}$. 
Quadro 1. Estudos sobre a correlação da ingestão e suplementação de cálcio e vitamina $D$ e risco de câncer colorretal

\begin{tabular}{|c|c|c|c|}
\hline Estudos & Tipos do estudo & $\begin{array}{l}\text { Número de } \\
\text { pacientes }\end{array}$ & Resultados \\
\hline Cho E et al. ${ }^{8}$ & $\begin{array}{l}\text { Meta-análise de } 10 \\
\text { estudos coortes }\end{array}$ & $\begin{array}{l}534.536 \text { indivíduos } \\
\text { com câncer } \\
\text { colorretal }\end{array}$ & $\begin{array}{l}\text { A ingestão }>250 \mathrm{~g} / \text { dia de leite está } \\
\text { relacionada com um menor risco de câncer } \\
\text { colorretal quando comparada com uma } \\
\text { ingestão }<70 \mathrm{~g} / \text { dia, e que o consumo de } \\
500 \mathrm{~g} / \text { dia está associado com a redução em } \\
12 \% \text { do risco de câncer colorretal }\end{array}$ \\
\hline Larsson SC et al. ${ }^{9}$ & Coorte & $\begin{array}{l}45.306 \text { homens } \\
\text { sem história de } \\
\text { câncer }\end{array}$ & $\begin{array}{l}\text { Alta ingestão de cálcio e produtos lácteos, } \\
\text { em especial o leite, está relacionada com } \\
\text { a diminuição do risco de câncer colorretal. } \\
\text { Além disso, observaram que homens que } \\
\text { consumiam } \geq 1,5 \text { copos de leite dia tinham } \\
33 \% \text { menos risco de desenvolver câncer } \\
\text { colorretal quando comparados com quem } \\
\text { consumia < } 2 \text { copos semana }\end{array}$ \\
\hline Jarvin $\mathrm{R}$ et al. ${ }^{10}$ & Coorte prospectivo & $\begin{array}{l}9.959 \text { indivíduos } \\
\text { de ambos os sexos } \\
\text { sem história prévia } \\
\text { de câncer }\end{array}$ & $\begin{array}{l}\text { Consumo de leite e produtos lácteos em } \\
\text { geral está inversamente relacionado com a } \\
\text { incidência do câncer colorretal. Além disso, } \\
\text { indivíduos que estavam no maior quartil } \\
\text { de consumo de leite tinham } 46 \% \text { menos } \\
\text { chance de desenvolver câncer de cólon, e } \\
\text { os que consumiam produtos láteos, } 37 \% \\
\text { menos chance. Entretanto esta associação } \\
\text { não aparece para ingestão total de cálcio, } \\
\text { vitamina D ou leite fermentado }\end{array}$ \\
\hline Hubner RA et al. ${ }^{11}$ & Estudo de prevenção & $\begin{array}{l}853 \text { indivíduos } \\
\text { com adenoma } \\
\text { colorretal }\end{array}$ & $\begin{array}{l}\text { Consumo de produtos lácteos está } \\
\text { inversamente relacionado com a reincidência } \\
\text { de adenoma colorretal, independente do } \\
\text { consumo de vitamina } D \text { e suplementação } \\
\text { de cálcio }\end{array}$ \\
\hline $\begin{array}{l}\text { McCullough ML et } \\
\text { al. }{ }^{12}\end{array}$ & Coorte & $\begin{array}{l}66.882 \text { mulheres } \\
\text { e } 60.866 \text { homens } \\
\text { sem câncer }\end{array}$ & $\begin{array}{l}\text { Houve uma modesta associação inversa } \\
\text { entre ingestão total de cálcio nos homens, } \\
\text { mas não nas mulheres. Contudo, sugerem } \\
\text { que doses moderadas de cálcio reduzem } \\
\text { o risco, mas altas doses não aumentariam } \\
\text { esses benefícios }\end{array}$ \\
\hline Terry $\mathrm{P}$ et al. ${ }^{13}$ & Coorte & 61.463 mulheres & $\begin{array}{l}\text { Observaram uma associação inversa entre } \\
\text { a ingestão de cálcio e câncer colorretal. } \\
\text { Mulheres com alta ingestão de cálcio (média } \\
\text { de } 914 \mathrm{mg} / \text { dia) tiveram redução do risco de } \\
\text { câncer colorretal quando comparadas com } \\
\text { mulheres com baixa ingestão ( } 486 \mathrm{mg} / \text { dia). } \\
\text { A ingestão de produtos lácteos foi fraca, } \\
\text { sugerindo que a ingestão de cálcio é mais } \\
\text { importante do que uma fonte específica de } \\
\text { cálcio. A ingestão da vitamina D não estava } \\
\text { claramente associada com o risco }\end{array}$ \\
\hline
\end{tabular}




\begin{tabular}{|c|c|c|c|}
\hline Estudos & Tipos do estudo & $\begin{array}{l}\text { Número de } \\
\text { pacientes }\end{array}$ & Resultados \\
\hline Park S et al. ${ }^{15}$ & Coorte & $\begin{array}{l}85.903 \text { homens e } \\
105.108 \text { mulheres }\end{array}$ & $\begin{array}{l}\text { Constataram uma inversa associação entre a } \\
\text { ingestão de produtos lácteos e leite e risco de } \\
\text { câncer colorretal. Ao analisar a ingestão de } \\
\text { cálcio total, observaram uma associação inversa } \\
\text { para o risco de câncer colorretal em ambos os } \\
\text { sexos, sendo que essa redução do risco foi de } \\
30 \% \text { para homens e } 36 \% \text { para mulheres }\end{array}$ \\
\hline Lin J et al. ${ }^{17}$ & Coorte prospectivo & 39.876 mulheres & $\begin{array}{l}\text { Não observaram associação com ingestão } \\
\text { e suplementação de vitamina } D \text { e risco } \\
\text { de câncer colorretal, eles acreditam que } \\
\text { esses resultados não significativos devam } \\
\text { ser atribuídos à dificuldade de mensurar } \\
\text { a ingestão de vitamina } D \text {, já que fontes } \\
\text { dietéticas de vitamina } D \text { são limitadas }\end{array}$ \\
\hline Martínez ME et al. ${ }^{18}$ & $\begin{array}{l}\text { Meta-análise de } 6 \\
\text { estudos coorte, } 18 \\
\text { estudos caso-controle } \\
\text { e } 1 \text { estudo coorte }\end{array}$ & $\begin{array}{l}1.012 .280 \\
\text { mulheres }\end{array}$ & $\begin{array}{l}\text { Não encontraram associação significativa } \\
\text { entre ingestão alimentar de cálcio e risco de } \\
\text { câncer colorretal. Também observaram fraca } \\
\text { associação inversa, porém não significativa } \\
\text { entre a ingestão dietética e ingestão total de } \\
\text { vitamina D e risco de câncer colorretal }\end{array}$ \\
\hline Kesse E et al. ${ }^{22}$ & Caso-controle & 5.495 indivíduos & $\begin{array}{l}\text { Suportam a hipótese de que a alta ingestão } \\
\text { de cálcio e produtos lácteos exerce efeito } \\
\text { protetor contra o desenvolvimento de } \\
\text { adenomas colorretais }\end{array}$ \\
\hline $\begin{array}{l}\text { Wactawski-Wende J } \\
\text { et al. }{ }^{23}\end{array}$ & $\begin{array}{l}\text { Estudo randomizado, } \\
\text { duplo cego e caso- } \\
\text { controle }\end{array}$ & 36.282 mulheres & $\begin{array}{l}18.176 \text { mulheres receberam } 1.000 \mathrm{mg} \text { de } \\
\text { cálcio e } 400 \text { UI de vitamina } \mathrm{D} \text {, e outras } \\
18.106 \text { receberam placebo durante } 7 \text { anos. } \\
\text { Estudo mostrou que a suplementação de } \\
\text { cálcio e vitamina D não afetou a incidência } \\
\text { de câncer colorretal entre essas mulheres }\end{array}$ \\
\hline
\end{tabular}

Mizoue et al. ${ }^{14}$, em estudo epidemiológico, descrevem o efeito protetor do consumo de cálcio e vitamina $\mathrm{D}$ para câncer colorretal em ocidentais, porém esses estudos são limitados na população asiática, a qual tem baixo consumo de cálcio. Investigaram a associação do consumo desses nutrientes nos japoneses através de um questionário dietético com 148 alimentos típicos da região. O estudo observou que a diminuição do risco de câncer colorretal com alta ingestão de cálcio foi observada entre as pessoas que tinham níveis elevados de ingestão de vitamina $\mathrm{D}$ ou entre os indivíduos que tinham exposição adequada ao sol.

Park et al. ${ }^{15}$, através de um estudo coorte realizado nos Estados Unidos, no período de 1993 a 1996, com 85.903 homens e 105.108 mulheres acima de 45 anos de idade, constataram uma inversa associação entre a ingestão de produtos lácteos e leite e risco de câncer colorretal. Ao analisar a ingestão de cálcio total, observaram uma associação inversa para o risco de câncer colorretal em ambos os sexos, sendo que essa redução do risco foi de 30\% para homens e 36\% para mulheres. É importante ressaltar que esse estudo ajustou a ingestão de gordura total, e a associação entre produtos lácteos com o risco de câncer colorretal não foi afetada.

Em outro estudo caso-controle, Slattery et al. ${ }^{16}$ mostraram que a alta ingestão de cálcio e vitamina $\mathrm{D}$ reduz o risco de desenvolvimento de câncer colorretal.

Em oposição aos resultados anteriores, um estudo coorte prospectivo realizado com mulheres acima de 45 anos, no período de 10 anos, não observou associação entre a ingestão alimentar e suplementação de cálcio com risco de câncer colorretal e; além disso, não acharam interação entre a ingestão de cálcio com ingestão de gorduras, fósforo, fibras e vitamina $\mathrm{D}$ em relação ao risco de câncer colorretal. Quando examinaram a ingestão de produtos lácteos, tais como: produtos lácteos fermentados, leite, queijo e iogurte, também não observaram associação significativa com o câncer colorretal $^{17}$. De acordo com esse resultado, uma meta-análise de seis estudos coorte e 18 estudos caso-controle e um estudo coorte no período de 12 anos com 1.012.280 mulheres ${ }^{18}$ não encontraram associação significativa entre ingestão alimentar de cálcio e risco de câncer colorretal. 
Norat et al. ${ }^{19}$ realizaram uma revisão dos estudos que associaram o consumo de produtos lácteos com câncer colorretal e observaram que os estudos caso-controle são heterogêneos, e que a grande maioria não achou evidências da associação entre a ingestão total de cálcio e produtos lácteos com o risco de câncer colorretal, entretanto os estudos coortes suportam a hipótese do efeito protetor de todos os produtos lácteos e leite especificamente, porém não observaram essa associação com o consumo exclusivo de queijo e iogurte.

Dois estudos randomizados avaliaram o efeito da suplementação do cálcio e o surgimento de adenomas colorretais e ambos observaram redução do risco ${ }^{20,21}$. No estudo de Bonithon-Kopp et al. ${ }^{20}$, foi observada uma redução de $30 \%$ do risco de desenvolvimento de adenomas após um ano de suplementação de $1.200 \mathrm{mg}$ de cálcio diariamente, sendo que o efeito protetor da suplementação ocorreu somente no grupo que tinha alta ingestáo dietética de cálcio. Baron et al. ${ }^{21}$ observaram que a ingestão de $2.000 \mathrm{mg}$, após três anos de suplementação de cálcio diariamente, estava associada com a diminuiçáo de 34\% do risco de desenvolver adenomas. Em um estudo caso-controle, com participação de 5.495 indivíduos, há a hipótese de que a alta ingestão de cálcio e produtos lácteos exerce efeito protetor contra o desenvolvimento de adenomas colorretais ${ }^{22}$.

Contrapondo estes últimos resultados, um estudo randomizado, duplo cego e caso-controle, envolvendo 36.282 mulheres; das quais, 18.176 mulheres receberam $1.000 \mathrm{mg}$ de cálcio e $400 \mathrm{UI}$ de vitamina D, e outras 18.106 receberam placebo durante sete anos, mostrou que a suplementação de cálcio e vitamina $\mathrm{D}$ não afetou a incidência de câncer colorretal entre essas mulheres ${ }^{23}$. Similarmente, Lin et al. ${ }^{17}$ não observaram redução do risco de câncer colorretal em grupos com ingestáo total de cálcio $\geq 1.350 \mathrm{mg}$ por dia.

Em alguns estudos com animais, o efeito protetor do cálcio no câncer colorretal foi mais pronunciado em animais alimentados com dietas ricas em gordura ${ }^{23}$. Nutrientes, tais como: fósforo e fibras podem reduzir a absorção intestinal de cálcio e consequentemente seus efeitos protetores do câncer coloretal ${ }^{24}$.

Park et al. ${ }^{15}$ mostraram que ingestáo total de vitamina D estava inversamente associada com risco de câncer colorretal em homens, mas não em mulheres; porém mulheres no maior quintil de ingestáo de vitamina $\mathrm{D}$ dietética mostrou uma redução significativa do risco relativo. Um estudo randomizado, duplo-cego e casocontrole constatou que a alta concentraçáo sérica de 25-hidroxivitamina D estava associada com efeitos protetores para adenomas colorretais ${ }^{25}$. Um estudo coorte que acompanhou 1.012.280 mulheres, no período de 12 anos, observou uma fraca associação inversa, porém não significativa entre a ingestáo dietética e ingestáo total de vitamina D e risco de câncer colorretal ${ }^{18}$. Outro estudo coorte prospectivo realizado com 39.876 mulheres não observou associaçáo com ingestáo e suplementação de vitamina $\mathrm{D}$ e risco de câncer colorretal, eles acreditam que esses resultados não significativos devam ser atribuídos à dificuldade de mensurar a ingestão de vitamina $\mathrm{D}$, já que fontes dietéticas de vitamina $\mathrm{D}$ são limitadas ${ }^{17}$.

\section{CÁLCIO E VITAMINA D E LOCALIZAÇÃO DO CÂNCER COLORRETAL}

Cho et al. ${ }^{8}$ constataram uma associação inversa entre os tumores de cólon (proximal e distal) e reto com o consumo de cálcio e produtos lácteos; além disso, observaram que o consumo de produtos lácteos variou de acordo com a localização, mostrando uma associação inversa do consumo de leite aos cânceres de cólon distal e reto; porém, na associação entre o consumo de queijo ou iogurte com a localização do câncer colorretal, não houve significância estatística.

Um estudo de meta-análise utilizou dados de 60 estudos epidemiológicos com 26.335 casos de câncer colorretal e observou que o alto consumo de cálcio teve um ótimo efeito protetor para tumores no cólon distal e reto versus cólon proximal. A redução do risco foi similar tanto para o consumo de produtos lácteos quanto para a suplementação de cálcio ${ }^{11}$.

Em contraste com os resultados anteriores, o Cancer Prevention Study II Nutrition Cohort ${ }^{12}$ constatou que uma alta ingestấo de cálcio total está relacionada com redução do risco de câncer colorretal proximal, mas não de cólon distal ou reto, e Gevaz et al. ${ }^{25}$ acharam uma associação inversa tanto para cólon proximal quanto cólon distal.

Alguns autores não encontraram associação entre cálcio e produtos lácteos com a localização do tumor. Larsson et al. ${ }^{9}$, através de um estudo coorte, mostraram que o risco relativo de câncer colorretal diminuía em homens que tinham alta ingestão de produtos lácteos quando comparados com baixa ingestáo, porém a associação entre consumo de produtos lácteos não diferiu entre localização do tumor.

É importante ressaltar que os produtos lácteos, além do cálcio, possuem outras substâncias que podem interferir diretamente na redução do risco do câncer colorretal. Os produtos lácteos possuem ácido linoleico conjugado e lactoferrina, os quais têm efeito inibitório da carcinogênese colônica em modelos animais ${ }^{10}$; e a caseína do leite tem uma atividade antimutagênica no trato digestivo ${ }^{12}$.

\section{CONCLUSÃO}

Está bem estabelecido que um dos fatores de risco para o desenvolvimento do câncer colorretal é a dieta. Entre os 
componentes da dieta, o cálcio e a vitamina D têm sido associados com o câncer colorretal.

Os estudos realizados se dividem nos resultados que relacionam o cálcio, produtos lácteos e vitamina $\mathrm{D}$ com o risco de desenvolvimento de câncer colorretal. Muitos estudos mostraram que alto consumo de produtos lácteos, principalmente o leite, está associado com a diminuição do risco.

Alguns estudos relacionaram a ingestão cálcio ou suplementaçáo com o risco do câncer colorretal, porém muitos deles não avaliaram a ingestão de alimentos que poderiam interferir na absorção do cálcio e consequentemente seus efeitos.

Os resultados do efeito da vitamina $\mathrm{D}$ e câncer colorretal foram controversos, isto se dá pela dificuldade de mensurar a ingestáo de vitamina $\mathrm{D}$ e pela síntese de vitamina $\mathrm{D}$ pelos raios ultravioletas, a qual é a principal fonte de vitamina $\mathrm{D}$, havendo necessidade de informaçôes sobre exposição do indivíduo ao sol.

Alguns estudos associaram o consumo de produtos lácteos, cálcio e vitamina $\mathrm{D}$ com a localização do câncer colorretal, os quais foram controversos. Sabe-se que a localizaçáo do desenvolvimento do câncer está relacionada com mudanças de fatores genéticos na oncogênese.

Existem evidências de que o cálcio e a vitamina $\mathrm{D}$ estáo relacionados com reduçáo da gênese do câncer colorretal.

\section{Declaração de Conflito de Interesses: Nada a Declarar REFERÊNCIAS}

1. Department of Helth and Human Services (USA). [homepage on the internet] National Institute. SEER cancer statistics review, 1975-2002:mortality-colon rectum, number of deaths by primary cancer site, race and sex, 1998-2002. (cited 2005 Apr 17). Availble from: URL:http://seer.cancer.gov/cgibin/csr/1975_2002/ searc-h.pl\#results.

2. Instituto Nacional de Câncer (Brasil). Estimativa 2010: Incidência de câncer no Brasil. Disponível em: URL:http://www.inca.gov.br/estimativa/2010.

3. Ströhle A. Nutrition and colorectal cancer. Med Monatsschr Pharm 2007;30:25-32.

4. Holt P. New insights into calcium, dairy and colon câncer. World J Gastroenterol 2008: 4429-4433.

5. Lamprecht SA, Lipkin M. Chemoprevention of colon cancer by calcium, vitamin $\mathrm{D}$ and folate: molecular mechanisms. Nat Rev Cancer 2003; 3: 601-14.

6. Peterlik M, Grant Wb, Cross Hs. Calcium, Vitamin D And Cancer. Anticancer Res 2009; 29:3687-98

7. Peter R. Dairy food and prevention of colon cancer: human studies. J Am Coll Nutr 1999; 18:37-91.
8. Cho E, Smith-Warner SA, Spiegelman D, et al. Dairy food, calcium, and colorectal cancer: a pooled analysis of 10 cohort studies. J Natl Cancer Inst 2004; 96: 1015-22.

9. Larsson SC, Bergkvist L, Ruteggard J, et al. Calcium and dairy food intake are inversely associated with colorectal cancer risk in the Cohort of Swedish men. Am J Clin Nutr 2006; 83:667-73.

10. Jarvinen $\mathrm{R}$ et al. Propective study on milk products, calcium and cancers of the colon and rectum. Eur J Clin Nutr 2001;55:1000-7.

11. Hubner RA, et al. Dairy products, polymorphisms in the vitamin $\mathrm{D}$ receptor gene and colorectal adenoma recurrence. Int J Cancer 2008; 123: 586-93.

12. McCullough ML, Robertson AS, Rodriguez C, et al. Calcium, vitamin D, dairy products, and risk of colorectal cancer in the Cancer Prevention Study II Nutrition Cohort (United States) Cancer Causes Control 2003; 14:1-12.

13. Terry $P$, et al. Dietary calcium and vitamin D intake and risk of colorectal cancer: a prospective cohort study in women. Nutr Cancer. 2002: 43: 39-46.

14. Mizoue T, et al. Calcium, dairy foods, vitamin D, and colorectal cancer risk: the Fukuoka Colorectal Cancer Study. Cancer Epidemiol Biomarkers Prev 2008:17:2800-7.

15. Park S, Murphy SP, Wilkens et al. calcium and vitamin $\mathrm{D}$ intake and risk of colorectal cancer: the multiethnic cohort study. American journal of epidemiology 2007; 165: 784-93.

16. Slattery ML et al. Dietary calcium, vitamin D, VDR genotype and colorectal cancer. Int J Cancer 2004;111: 750-6.

17. Lin J, Zhang SM, Cook NR, et al. Intake of calcium and vitamin $\mathrm{D}$ and risk of colorectal cancer in woman. American journal of epidemiology 2005; 161:755-64.

18. Martínez ME, Giovannucci EL, Colditz GA, et al. Calcium, vitamin $\mathrm{D}$, and the occurrence of colorectal cancer among women. Natl Cancer Inst 1996;88:1375-82.

19. Norat $T$ et al. Dairy products and colorretal cancer. A review of possible mechanisms and epidemiological evidence. Eur J Clin Nutr. 2003: 57: 1-17.

20. Bonithon-Kopp C, Kronborg O, Giacosa A, et al. Calcium and fibre supplementation in prevention of colorectal adenomas recurrence: a randomised intervention trial. European Cancer Prevention Organisation Study Group. The Lancet. 2000; 356:1300-6.

21. Baron JA, Beach M, Mandel JS et al. Calcium supplements for the prevention Study Group. N Engl J Med 1999;340:101-7.

22. Kesse E et al. Dietary calcium, phosphorus, vitamin $\mathrm{D}$, dairy products and the risk of colorectal adenoma and cancer among French women of the E3N-EPIC prospective study. Int J Cancer 2005;117:137-44. 
23. Wactawski-Wende J et al. Calcium plus vitamin D supplementation and the risk of colorectal cancer. $\mathrm{N}$ Engl J Med 2006; 354:684-96.

24. Grau MV, Baron Jam Sandler RS, et al. Vitamin D, Calcium supplementation, and colorectal adenomas
Results of a randomized trial. J Natl Cancer Inst 2003; 95:1765:71.

25. Gervaz P, Bucher P, Morel P et al. Two colons-two cancer: paradigm shift and clinical implication. J Surg Oncol 2004;88:261-6.

\section{Abstract}

Colorectal cancer is the third most prevalent neoplasm type in the world. The main risk factors are: familiar history of colon and rectum cancer, diet on the basis of animal fats, low intake of fruits, vegetables and cereals, excessive consumption of alcohol and tobacco and advanced age. Evidence from animal studies has suggested that calcium and vitamin $\mathrm{D}$ intake may reduce colonic carcinogenesis. The objective is to discuss the association between calcium and vitamin D intake and colorectal cancer risk. A bibliographic review based on articles published within the last 10 years, from magazines indexed in virtual libraries such as Lilacs, SciELO and Medline was done. One of the mechanisms in which calcium reduces colorectal cancer risk is its ability to bind itself to bile acids and ionized fatty acids, thereby reducing the potential proliferation effects of these compounds on the colonic mucosa. Vitamin D has the potential to regulate cell proliferation. Studies have shown that high consumption of milky products, mainly milk, is associated with risk reduction. There are evidences that calcium and vitamin D are related with genesis reduction of colorectal cancer.

Key words: Colorectal Neoplasms; Calcium; Vitamin D; Dairy Products; Review

\section{Resumen}

El cáncer colorrectal es el tercer tipo de neoplasia más prevalente de cáncer. Algunos factores de riesgo son: histórico familiar de cáncer de colon y recto, alimentación basada en grasas animales, bajo consumo de frutas, hortalizas y cereales, consumo excesivo de alcohol y tabaco, además de edad avanzada. La evidencia de estudios animales ha sugerido una posible actuación de calcio y algunas vitaminas D como anticarcinógenos colorrectales. El objetivo es discutir la relación entre la ingesta alimentar y suplementación del calcio y vitamina D y el riesgo de cáncer colorrectal. Se ha hecho una revisión bibliográfica de artículos publicados en los diez últimos ańos, de fuentes de revistas indexadas en las bibliotecas virtuales Lilacs, SciELO y Medline. Uno de los mecanismos por el cual el calcio reduce el riesgo de cáncer colorrectal es su capacidad de unirse a las sales biliares y a los ácidos grasos ionizados, lo que reduce el efecto de compuestos de proliferación celular de la mucosa colónica. La vitamina D regula la proliferación celular. Los estudios habían demostrado que el alto consumo de productos lechosos, principalmente la leche, se asocia a la reducción del riesgo. Hay evidencias de que el calcio y la vitamina $\mathrm{D}$ estén relacionados a la reducción de la génesis del cáncer colorrectal.

Palabras clave: Neoplasias Colorrectales; Cálcio; Vitamina D; Productos Lácteos; Revisión 